Sharif University of Technology
Scientia Iranica
SCIENTIA

\title{
Transient behavior of electrostatically actuated micro systems considering squeeze film damping and mechanical shock
}

\author{
J. Abderezaei and M. Moghimi Zand* \\ School of Mechanical Engineering, College of Engineering, University of Tehran, Tehran, P.O. Box 14399-55961, Iran.
}

Received 6 December 2015; received in revised form 11 September 2016; accepted 29 October 2016

\author{
KEYWORDS \\ MEMS; \\ NEMS; \\ Nonlinearity; \\ Electrostatic \\ actuation; \\ Newmark method; \\ Pull-in instability; \\ Fringing field.
}

\begin{abstract}
In this paper, the dynamics of microbeams under the effects of electrostatic force, mechanical shock, Squeeze Film Damping (SQFD), and fringing field are modeled. A Galerkin-based reduced-order model is used to convert the Partial Differential Equation of motion (PDE) to an Ordinary Differential Equation (ODE). Furthermore, the system dynamics are studied using the developed nonlinear finite element code. Two different simpler models are validated by the results in the literature, which are in good compatibility with them. It is shown that the effect of squeeze film damping can dominate mechanical shock significantly. The response of microbeam to electrostatic actuation is also delayed when damping is included. The simultaneous and sole effects of electrostatic actuation, mechanical shock, squeeze film damping, and fringing field are investigated in this study for the first time.
\end{abstract}

(C) 2017 Sharif University of Technology. All rights reserved.

\section{Introduction}

The clamped-clamped microbeam is an essential structural component in MEMS and plays a substantial role in many RF-Microelectromechanical Systems (MEMS). The introduction of this component led to a revolution in the production of various mechanical and electrical devices. With the increasing applications of MEMS in our lives, and their abrupt growth in today's industries as sensors, actuators, RF filters, switches, and microwave signal processors integrated with electronics, their role has become even more important. Electrostatically actuated MEMS are used in a variety of different tools and applications such as capacitive

\footnotetext{
*. Corresponding author. Tel.: +982161114807; Fax: +982188013029

E-mail address: mahdimoghimi@ut.ac.ir (M. Moghimi Zand)
}

doi: $10.24200 /$ sci. 2017.4245 and optical switches, pressure and inertial sensors, resonators, and accelerometers. They are also useful in radio frequency applications and micro/nano mechanical memories. In addition, they can experience mechanical shocks during fabrication procedure, assembly process, and transportation. When electrostatically actuated MEMS are under the effect of electrostatic force, the microbeam moves toward substrate; consequently, the fluid underneath the beam has a massive movement and the so called Squeeze Film Damping (SQFD) will reduce the system vibrations. All the above effects have significant influences on MEMS behavior; consequently, in this paper, these effects are investigated.

The electrostatically actuated MEMS have been studied by some researchers. Nathanson et al. [1] studied a simplified microbeam, mechanically and electrically. They derived an expression for characteristic voltage, which was called pull-in voltage, that could lead to instability in micro/nano systems. Taylor [2] also reported and investigated pull-in phenomenon in an electrical field. Zhang et al. [3] presented an 
overview of pull-in phenomenon in micro/nano systems. To do so, they studied various conditions and physical principles that could lead to static and dynamic instabilities. Farrokhabadi et al. [4] studied effects of Casimir force on pull-in instability of nanosystems. They employed Euler-Bernoulli beam theory and couple stress continuum theory to derive governing equations. Rochus et al. [5] investigated dynamic pullin instability of a microsystem using finite element approach. Ahn et al. [6] designed electrostatically driven microbeam resonators. Seshia et al. [7] used a novel analytical model to investigate the performance of a vacuum packaged resonant accelerometer. Ko and Qiang [8] discussed design and characteristics of touchmode pressure sensors.

There are also several papers focusing on shock loads, with or without imposing electrostatic force. Brown [9] described harsh environments and checked both high-g shock and low-g shock MEMS. Tas et al. [10] used different methods to minimize possibility of stiction, caused by electrostatic forces and mechanical shocks. Li et al. [11] studied shock impact on MEMS microphones. Sheehy et al. [12] analyzed microcantilevers under vibrations and shocks by carrying out tests on a modified Hopkinson pressure bar and a vibration table. Kimberley et al. [13] investigated dynamic failure of Au RF-MEMS by conducting three different experiments with different ranges of loads. Srikar and Senturia [14] investigated the mechanical response of a microstructure and its elastic substrate under a large amount of shock pulses. They investigated the possibility of damages to the substrate as well as failure criteria of the microstructure. $\mathrm{Li}$ and Shemansky [15] investigated the result of dropping a microstructure on a solid ground. They modeled the microsystem, analytically and numerically, and showed an agreement between both solutions.

Squeeze film damping has been discussed in several papers; although squeeze film damping has been discussed in several papers, there are few studies focusing on MEMS behavior under shock impact, electrostatic force, and SQFD effect simultaneously. As an early research, Krylov and Maimon [16] analyzed the dynamic behavior of a microbeam. In their study, in addition to electrostatic force, they considered squeeze film damping and rotary inertia. Krylov [17] also investigated the dynamic pull-in instability of microbeams while considering nonlinear squeeze film damping. McCarthy et al. [18] used time-transient finite difference analysis to investigate dynamic behavior of a microswitch. Younis [19] studied effects of squeezefilm damping, thermoelastic damping, and structural forces in an electrostatically actuated MEMS system and, to do so, he utilized a hybrid numerical-analytical solution. Moghimi Zand and Ahmadian [20] studied dynamic behavior of an electrostatically actuated mi- crobeam while considering in-plane forces. They used First-order Shear Deformation Theory (FSDT) and applied Hybrid FEM-FDM to solve plate equations and Reynolds equation of squeeze film damping, simultaneously. Nayfeh and Younis [21] modeled the effect of squeeze film damping on a plate, using Reynolds equation to investigate a microstructure.

There are a number of differences in the behavior of systems in microscale and macroscale. Therefore, the scaling effects should be considered in microscale. Younis and Nayfeh [22] simulated the squeeze film damping in microplates while considering large electrostatic loads. Tajalli et al. [23] studied the dynamic pull-in effect of microplates, which was the result of a suddenly applied electrostatic force, while considering different effects of nonlinearity, fluid pressure, and various geometric parameters. For further studies on scaling effects in MEMS/NEMS, please see Refs. [2433 .

The present paper aims to investigate the transient response of clamped-clamped microbeams under the effects of electrostatic force, mechanical shock, squeeze film damping, and fringing field. For this purpose, we developed a finite element model using Galerkin's procedure; afterwards, we used Newmark time discretization method to derive dynamic responses. After validating the method and results, we present different diagrams to compare the effects of various parameters.

\section{Modeling and formulation}

Here, we consider a clamped-clamped microbeam, suspended over a fixed rigid electrode (substrate). As shown in Figure 1, the length of microbeam is $L$, width is $b$, density is $\rho$, thickness is $h, E$ is Young's modulus, the initial air gap is $d$, and $x$ is the coordinate along the length of the microbeam (Figure 1). The shock load is exerted on the microbeam through its supports [34]. This modeling is equivalent to applying the shock as a distributed force on the microstructure. We consider a microbeam exposed to a mechanical shock force $\left(F_{s h}\right)$ per unit length of amplitude $F_{0}$ and shape $g(t)$. The shock force in the supports is in the form of an

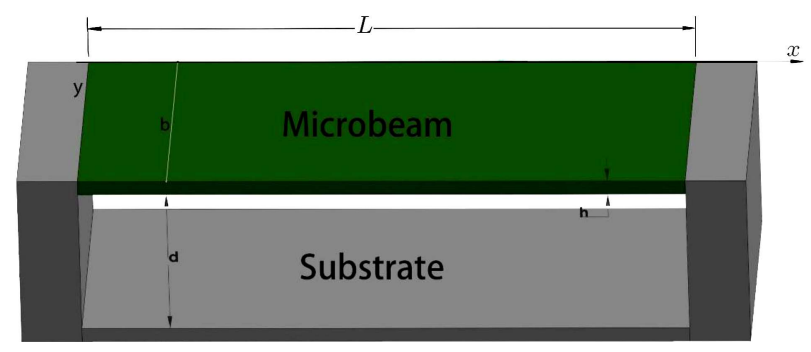

Figure 1. Schematic of a clamped-clamped microbeam suspended over a substrate. 
acceleration pulse of magnitude $a$, which is related to $F_{0}$ by $F_{0}=a \rho b h$. The mechanical shock is considered to be half-sine and can be described mathematically as:

$$
g(t)=\sin \left(\frac{\pi}{T} t\right)\{\hat{H}(t)-\hat{H}(t-T)\},
$$

where $\hat{H}(t)$ is the Heaviside function.

The microbeam is actuated by an electrostatic load of voltage, V, combined with a shock. After microbeam deflection, due to SQFD effect, microbeam experiences a backward pressure. In this research, the effect of electrostatic loading is considered as a step-input. For different types of electrostatic loading, please see ref. [35].

Generally, a microbeam can be modeled by the Euler-Bernoulli beam equation combined with an electrostatic actuation, a mechanical shock, and the nonlinear mid-plane stretching. The squeeze film damping can be modeled by the nonlinear Reynolds equation [36]. Therefore, the equation of beam motion would be:

$$
\begin{aligned}
E I \frac{\partial^{4} w}{\partial x^{4}} & +\rho b h \frac{\partial^{2} w}{\partial t^{2}}+C_{s q} \frac{\partial w}{\partial t} \\
= & {\left[\frac{E b h}{2 L} \int_{0}^{L}\left(\frac{\partial w}{\partial x}\right)^{2} d x+\tilde{N}\right] \frac{\partial^{2} w}{\partial x^{2}} } \\
& +\frac{1}{2} C_{r} \frac{\varepsilon b V^{2}}{(d-w)^{2}}+F_{0} g(t),
\end{aligned}
$$

where $\varepsilon$ is the dielectric constant of the gap medium, $I$ is the beam moment of inertia, and the parameter $\tilde{N}$ is the axial load; the fringing field parameter is:

$$
\begin{gathered}
C_{s q}=\frac{\bar{C}_{s q}}{(1-w / d)^{3}}, \\
\bar{C}_{s q}=\frac{\mu}{r^{3}} ; \quad r=\frac{d}{b},
\end{gathered}
$$

where $\mu$ is Viscosity coefficient of air. Here, $C_{r}$ is the fringing-field correcting capacitance factor, which is defined to represent the fringing field effect [37]

$$
\begin{array}{ll}
C_{r}=1+4.246 r, & 0 \leq r \leq 0.005 \\
C_{r}=1+\sqrt{11.0872 r^{2}+0.001097}, & 0.005 \leq r \leq 0.05 \\
C_{r}=1+1.9861 r^{0.8258}, & 0.05 \leq r
\end{array}
$$

For the sake of convenience, we introduce nondimensional variables,

$$
\hat{x}=\frac{x}{l}, \quad \hat{t}=\frac{t}{\tilde{t}}, \quad \hat{w}=\frac{w}{d},
$$

where $\tilde{t}=\sqrt{\frac{\rho b h l^{4}}{E I}}$ is the time scale. Substituting Eq. (6) into Eq. (2) and dropping the hats, we obtain:

$$
\begin{gathered}
\frac{\partial^{4} w}{\partial x^{4}}+\frac{\partial^{2} w}{\partial t^{2}}+\frac{C}{(1-w)^{3}} \frac{\partial w}{\partial t}=\left[\alpha_{1} \int_{0}^{L}\left(\frac{\partial w}{\partial x}\right)^{2} d x\right. \\
+N] \frac{\partial^{2} w}{\partial x^{2}}+\alpha_{2} C_{r} \frac{V^{2}}{(1-w)^{2}}+\alpha_{3} g(t),
\end{gathered}
$$

where the non-dimensional parameters are:

$$
\begin{aligned}
& C=\frac{\bar{C}_{s q} L^{4}}{E I \tilde{t}}, \quad \alpha_{1}=6\left(\frac{d}{h}\right)^{2}, \quad \alpha_{2}=\frac{6 \varepsilon L^{4}}{E h^{3} d^{3}} \\
& \alpha_{3}=\frac{12 L^{4} F_{0}}{E b d h^{3}}, \quad N=\frac{12 \tilde{N} L^{2}}{E b h^{3}} .
\end{aligned}
$$

In order to develop a finite element model, the variable $W(x, t)$ is approximated, utilizing the Hermite interpolation functions as:

$$
w(x, t)=\sum_{i=1}^{M} u_{i}(t) \phi_{i}(x),
$$

where $u_{i}(t)$ is the $i$ th generalized coordinate and $\phi_{i}$ are the Hermite interpolation functions. The cubic Hermite interpolation functions in terms of the element coordinates $\zeta$ are expressed as [38]:

$$
\left\{\begin{array}{l}
\phi_{1}(\zeta) \\
\phi_{2}(\zeta) \\
\phi_{3}(\zeta) \\
\phi_{4}(\zeta)
\end{array}\right\}=\left\{\begin{array}{c}
1-3 \zeta^{2}+2 \zeta^{3} \\
-\zeta(1-\zeta)^{2} \\
\zeta^{2}(3-2 \zeta) \\
-\zeta^{2}(\zeta-1)
\end{array}\right\}
$$

To analyze the dynamic response of the microbeam, we use a reduced order formulation based on Galerkin's procedure. Using Eq. (9) in the described weak form equations, we can develop the semi-discrete finite element model of Eq. (7) as:

$$
\left[M^{e}\right]\{\ddot{w}\}+\left[c^{e}\right]\{\dot{w}\}+\left[K^{e}\right]\{w\}=\left\{F^{e}\right\},
$$

where $\left[M^{e}\right],\left[K^{e}\right],\left[C^{e}\right]$, and $\left\{F^{e}\right\}$ are the mass matrix, the stiffness matrix, the damping matrix, and the force vector of element, respectively, and their coefficients are defined as:

$$
\begin{aligned}
& M_{i j}=\int_{\Omega^{e}} \phi_{i} \phi_{j} d x, \quad K_{i j}=\int_{\Omega^{e}}\left(\phi_{i} \phi_{j}+\alpha_{1} N_{s t} \phi_{i}^{\prime} \phi_{j}^{\prime}\right) d x, \\
& C_{i j}=\int_{\Omega^{e}} \frac{C}{(1-w)^{3}} \phi_{i} \phi_{j} d x, \quad F_{i}=F_{s h}+F_{e s}, \quad(12)
\end{aligned}
$$

where:

$$
\begin{aligned}
& N_{s t}=\int_{0}^{1}\left(\frac{\partial w}{\partial x}\right)^{2} d x, \quad F_{s h}=\int_{\Omega^{e}} \alpha_{3} g(t) \phi_{i} d x, \\
& F_{e s}=\int_{\Omega^{e}} \alpha_{2} C_{r} \frac{V^{2}}{(1-w)^{2}} \phi_{i} d x .
\end{aligned}
$$


The finite element model of the whole beam is derived by assembling the equations of all elements. Afterward, the fully discretized form of the problem is found using Newmark time discretization [38]. There are several procedures for Newmark method. In this paper, we implement the constant-average acceleration method.

\section{Results and discussions}

In order to validate the finite element model, our results are compared with the data presented in the literature. In Figure 2, we validate the model considering shock and electrostatic force, ignoring damping effect with the model in [39]. The comparison is performed using a clamped-clamped microbeam considering various properties: $L=900 \mu \mathrm{m}, b=100 \mu \mathrm{m}, d=2 \mu \mathrm{m}$, $h=1.5 \mu \mathrm{m}, E=169 \mathrm{GPa}$, and $\rho=2332 \mathrm{Kg} / \mathrm{m}^{3}$ The microbeam is actuated by $V=1.96 \mathrm{~V}$ and the mechanical shock amplitude of $1000 \mathrm{~g}$ with duration of $T=1 \mathrm{~ms}$. It can be seen that our results are in good agreement with the literature. As shown in Figure 2, microbeam undergoes large deflections near substrate; while it does not touch the substrate and, as the shock duration finishes, the microbeam fluctuates around an approximately constant position .

In Figure 3, to further validate our model, we compare results of the damped microbeam with the results in [40]. To perform the comparison, we use the same properties and the same shock amplitude as before, and ambient pressure of $P_{a}=10^{3} \mathrm{~Pa}$, while we ignore the electrostatic actuation. It is shown that our calculated results are in agreement with the literature. As seen in this figure, because of the SQFD, there are no secondary oscillations during the shock and the

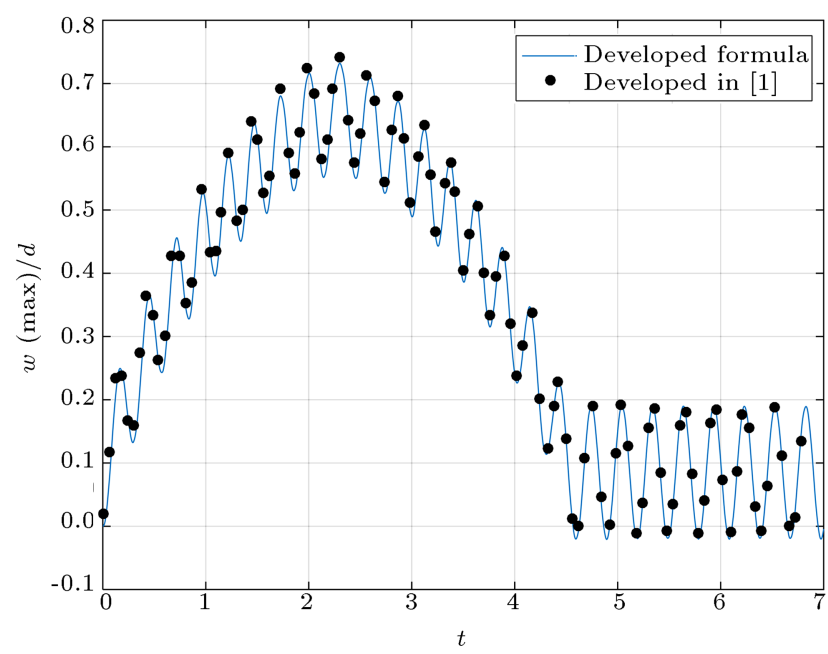

Figure 2. The normalized maximum amplitude of a microbeam subjected to a $1000 \mathrm{~g}$ shock pulse of $T=1.0 \mathrm{~ms}$ and $V=1.96 \mathrm{~V}$ versus the non-dimensional time.

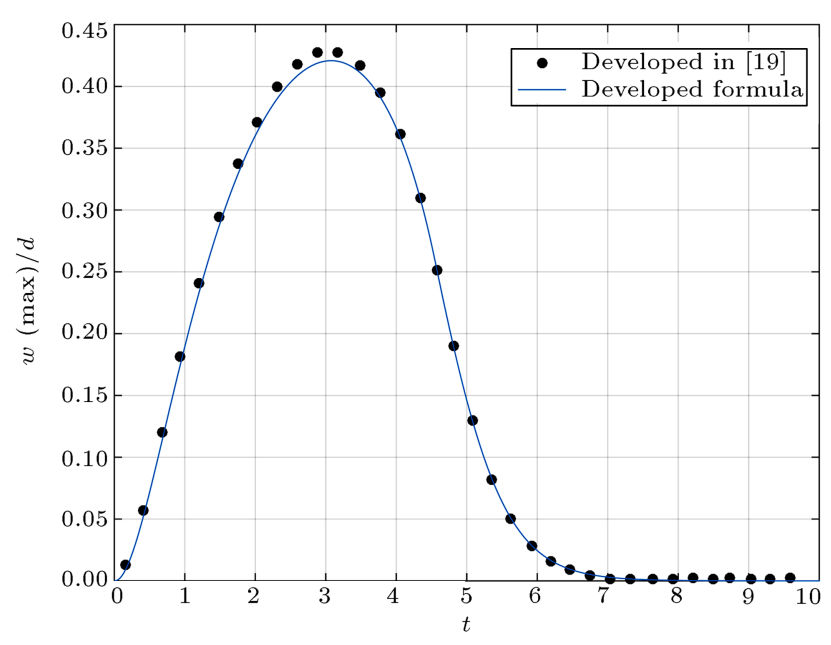

Figure 3. Time history of the microbeam under $1000 \mathrm{~g}$ shock pulse of $T=1.0 \mathrm{~ms}$ while considering SQFD and $V=0 \mathrm{~V}$.

maximum deflection of the microbeam is reduced as compared to the no-SQFD condition.

Figure 4 illustrates the time history of microbeam considering various mechanical shock durations. In Figure 4(a), we present dynamic responses with and without damping and fringing field effect. It is clear that by ignoring damping effect, the microbeam touches the substrate; however, when damping effect is considered, the microbeam does not touch the substrate and it undergoes a large deformation toward substrate until the shock duration finishes. It can be seen that the squeezed fluid between the microbeam and the substrate has a noticeable effect on the dynamic behavior of the microbeam. Therefore, for more accurate modeling, it is substantial to take this effect into account. In Figure 4(b), we change shock duration to $T=0.1 \mathrm{~ms}$. It is seen in Figure $4(\mathrm{a})$ and (b) that as fringing field is considered, there is an increase in maximum deflection for both shock durations; however, it is clear that this effect is more considerable in a shock duration of $T=1.0 \mathrm{~ms}$. When there is no fringing field, system stiction is delayed in no-SQFD case. Also, when SQFD exists, microbeam deflection is much higher. It is also noteworthy that fringing field effect is more significant when the shock duration is finished and there is only electrostatic actuation.

In Figure 5, we study the individual and simultaneous effects of shock and electrostatic force while considering the SQFD effect. First, we only consider a shock without imposing any voltage; then, we only include an electrostatic actuation. The third case shows the effects of both electrostatic actuation and shock load simultaneously.

Figure 6 shows the response of microbeam at $a=1000 \mathrm{~g}$ and $V=1.66 \mathrm{~V}$ for various initial gaps. When there is no damping, as $d$ increases, $W_{c} / d$ decreases; however, when the damping is included, 


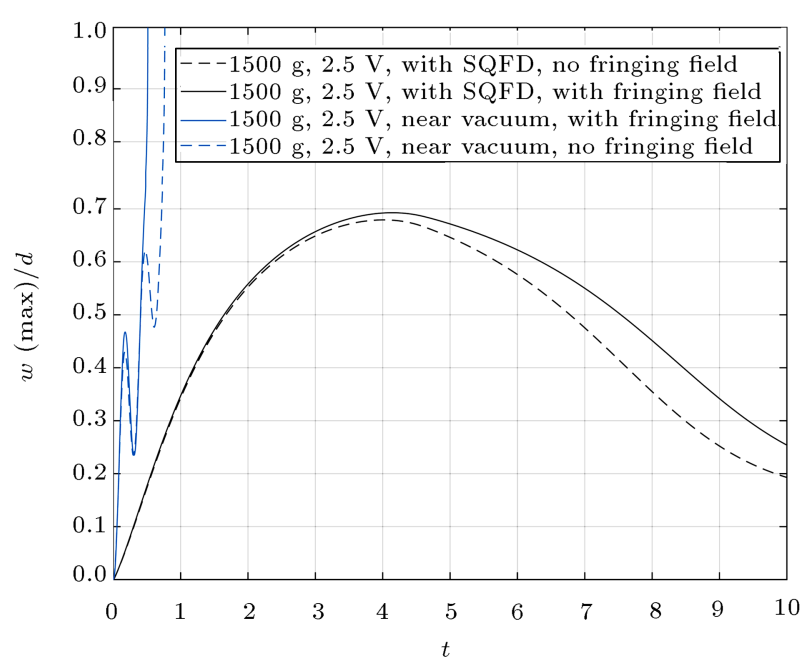

(a)

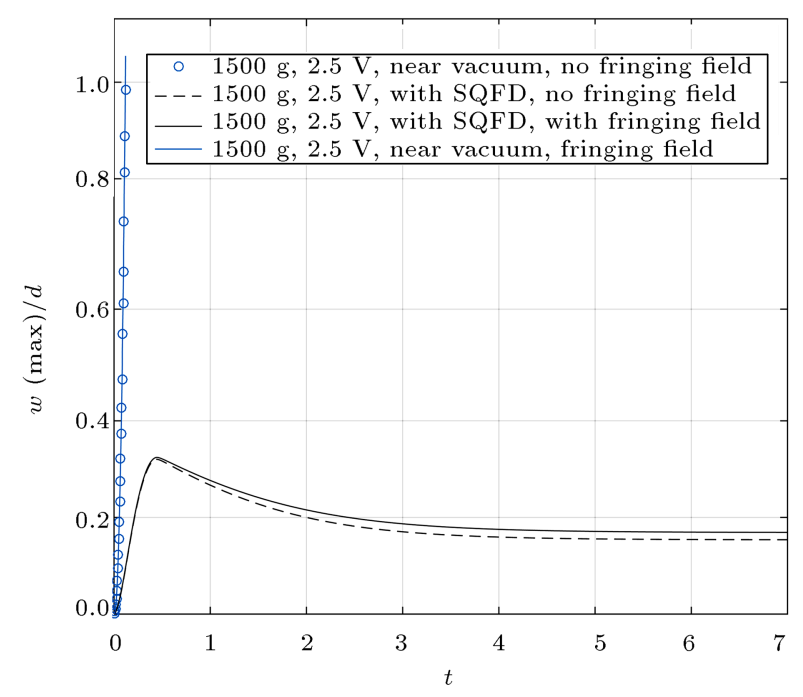

(b)

Figure 4. Time history of the microbeam under different amplitudes of shock and voltage: (a) $T=1.0 \mathrm{~ms}$, and (b) $T=0.1 \mathrm{~ms}$.

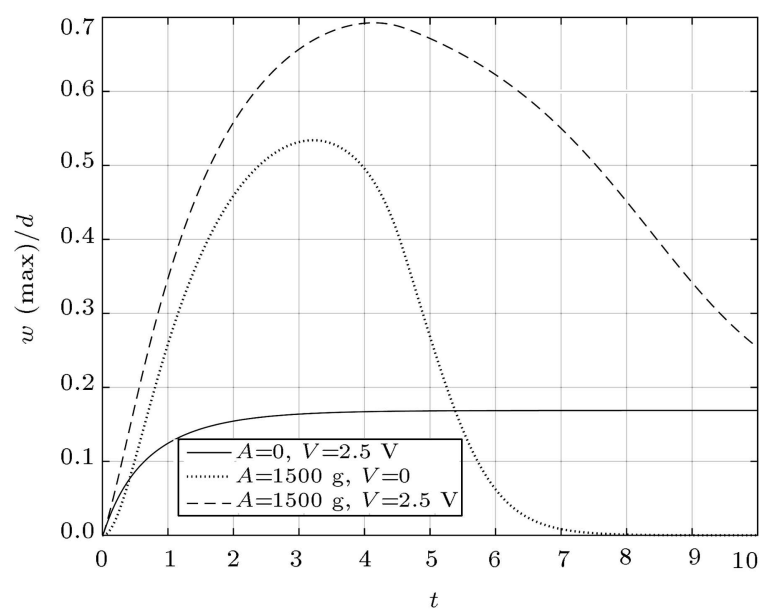

Figure 5. Time history of the microbeam under each force, individually and simultaneously.

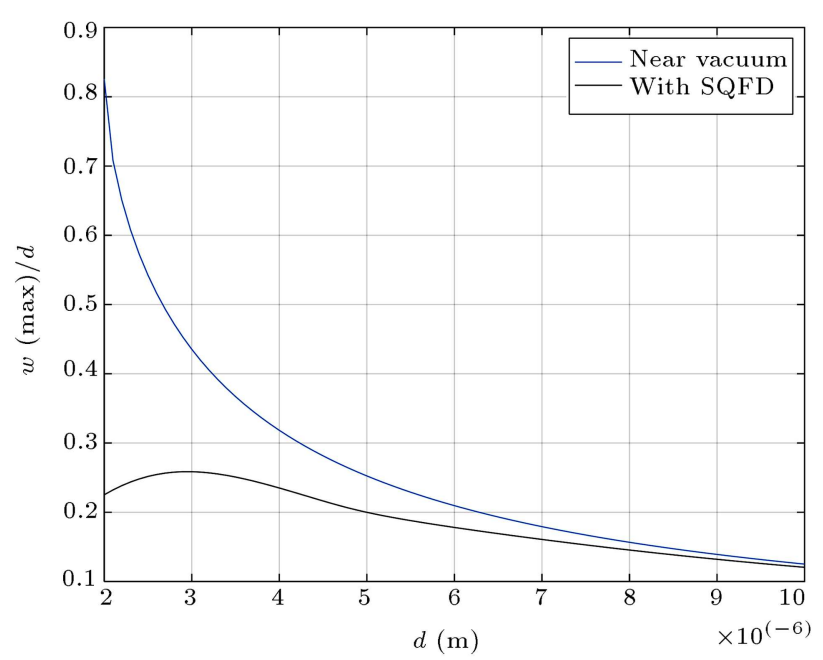

Figure 6. The maximum midpoint deflection versus initial gap width under shock amplitude of $1000 \mathrm{~g}$ and $V=1.66 \mathrm{~V}$ with and without SQFD.

the behavior of the system changes significantly and it shows two distinct trends. First, by increasing $d$ to a specific value, $W_{c} / d$ increases due to the SQFD effect. When $d$ is small, the effect of SQFD is significant and this results in smaller $W_{c} / d$. As $d$ increases to a certain amount, the built-up pressure (the result of SQFD), which is the main reason for smaller $W_{c} / d$, is relieved, and by increasing $d$ even more, the trend shows similar behavior to that in the near-vacuum condition; therefore, we can conclude that after a specific value of $d$, SQFD does not have a considerable effect on the microbeam behavior.

For design purposes, it is important to know maximum $W_{c} / d$ for various voltages and mechanical shocks; thus, in Figure $7(\mathrm{a})$, we study microbeam maximum deflection while considering SQFD and fringing field effect. In this figure, the shock amplitude changes from 0 to $2500 \mathrm{~g}$, the input voltage is from 0 to 2.5 volts, and the shock duration is $T=1.0 \mathrm{~ms}$. It is clear that by increasing both shock amplitude and voltage, maximum $W_{c} / d$ increases. It is shown that increasing the electrostatic actuation results in greater $W_{c} / d$. It also shows that SQFD dominates the shock impact significantly. Figure $7(\mathrm{~b})$ shows maximum $W_{c} / d$ for shock amplitudes from 0 to 9800 $\mathrm{g}$ and input voltage from 0 to 3 volts (shock duration is $T=0.1 \mathrm{~ms})$. It shows that increasing the shock amplitude and voltage results in the stiction of the microbeam. It is also shown that a shock duration of $T=1 \mathrm{~ms}$ leads to a larger deflection in comparison with the duration of $T=0.1 \mathrm{~ms}$. In Figure 7(a), increasing the shock to more than $2500 \mathrm{~g}$ and input voltage to more than 2.5 volts results in the stiction of microbeam; however, in Figure 7(b), these amounts for shock amplitude and input voltage are $9800 \mathrm{~g}$ and 3 volts, respectively. Additionally, it is noteworthy that 


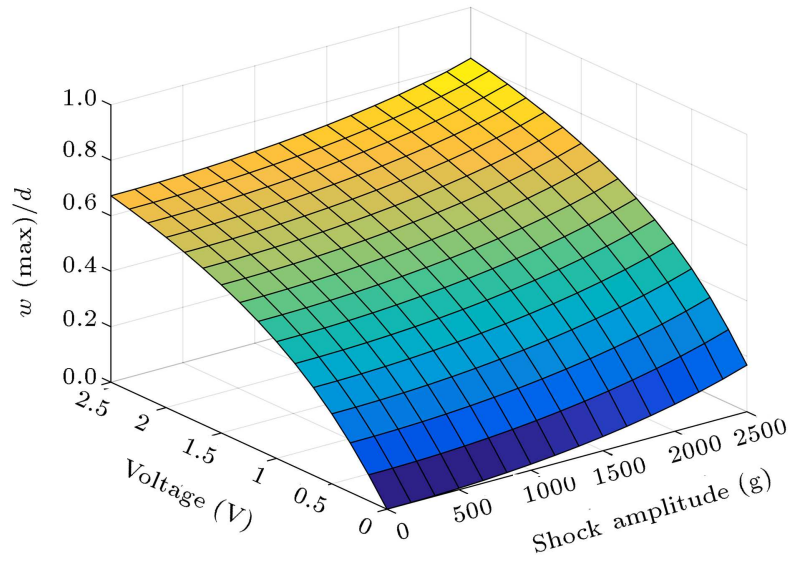

(a)

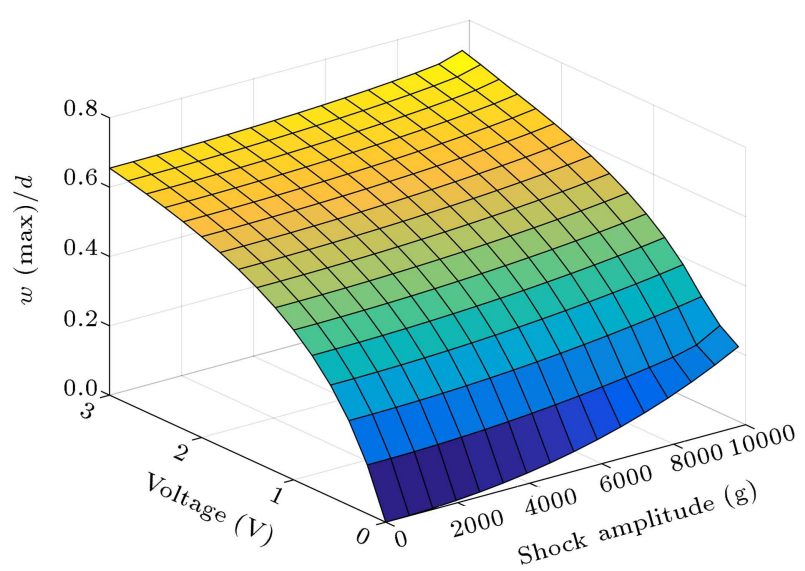

(b)

Figure 7. The maximum mid-point deflection for different shock amplitudes and different voltages for shock durations of (a) $T=1.0 \mathrm{~ms}$ and (b) $T=0.1 \mathrm{~ms}$.

in the $T=0.1 \mathrm{~ms}$ case, at some specific amplitudes, effect of the electrostatic actuation overcomes the shock effect.

\section{Conclusions}

In this paper, we presented modeling, simulation, and characterization of a microbeam under the effects of mechanical shock, electrostatic actuation, SQFD, fringing field, and geometric nonlinearity. For this purpose, we utilized FEM and Newmark time integration method to solve the problem. The responses of microbeams under the shock load and electrostatic actuation were validated by the literature.

Due to existence of the mid-plane stretching, the shock response of the microbeam is nonlinear. Therefore, when an electrostatic actuation is applied, it could result in an early dynamic pull-in instability. However, when the SQFD is considered, it is shown that the microbeam deflection is reduced significantly and the dynamic pull-in instability is delayed considerably. When the effect of SQFD was studied for the shock impact and electrostatic actuation individually, it was seen that the SQFD could dominate the mechanical shock, noticeably, while for the electrostatic actuation, the microbeam deflection was delayed when the SQFD was considered. Additionally, it was seen that there was no secondary oscillations when the SQFD effect was considered. The effects of initial gap, shock, electrostatic actuation, SQFD, and fringing field were studied, individually and simultaneously, in order to present appropriate information on the desirable applications. The results are believed to be useful in MEMS design.

\section{Acknowledgment}

The authors would like to thank Iranian National Science Foundation (INSF) for their support.

\section{References}

1. Nathanson, H.C., Newell, W.E., Wickstrom, R.A. and Davis, J.R. "The resonant gate transistor," IEEE Transactions on Electron Devices, 14(3), pp. 117-133 (1967).

2. Taylor, G. "The coalescence of closely spaced drops when they are at different electric potentials," in Proceedings of the Royal Society of London A: Mathematical, Physical and Engineering Sciences, pp. 423434 (1968).

3. Zhang, W.-M., Yan, H., Peng, Z.K. and Meng, G. "Electrostatic pull-in instability in MEMS/NEMS: A review", Sensors and Actuators A: Physical, 214, pp. 187-218 (2014).

4. Farrokhabadi, A., Mokhtari, J., Rach, R. and Abadyan, A. "Modeling the influence of the Casimir force on the pull-in instability of nanowire-fabricated nanotweezers", International Journal of Modern Physics B, 29(02), p. 1450245 (2015)

5. Rochus, V., Rixen, D.J. and Golinval, J.C. "Electrostatic coupling of MEMS structures: transient simulations and dynamic pull-in", Nonlinear Analysis: Theory, Methods and Applications, 63(5), pp. e1619e1633 (2005).

6. Ahn, Y., Guckel, H. and Zook, J.D. "Capacitive microbeam resonator design", Journal of Micromechanics and Microengineering, 11(1), p. 70 (2001).

7. Seshia, A., Palaniapan, M., Roessig, T., Howe, R.T, Gooch, R.W., Schimert, T.R. et al. "A vacuum packaged surface micromachined resonant accelerometer", Microelectromechanical Systems, Journal of, 11(6), pp. 784-793 (2002).

8. Ko, W.H. and Wang, Q. "Touch mode capacitive pressure sensors", Sensors and Actuators A: Physical, 75(3), pp. 242-251 (1999).

9. Brown, T.G. "Harsh military environments and microelectromechanical (MEMS) devices", in Sensors, Proceedings of IEEE, pp. 753-760 (2003). 
10. Tas, N., Sonnenberg, T., Jansen, H., Legtenberg, R. and Elwenspoek, M. "Stiction in surface micromachining", Journal of Micromechanics and Microengineering, 6(4), p. 385 (1996).

11. Li, J., Makkonen, J., Broas, M., Hokka, J., Mattila, T., Paulasto-Krockel, M. et al. "Reliability assessment of a MEMS microphone under shock impact loading", in Thermal, Mechanical and Multi-Physics Simulation and Experiments in Microelectronics and Microsystems (EuroSimE), 14th International Conference on, pp. 16 (2013).

12. Sheehy, M., Punch, J., Goyal, S., Reid, M. Lishchynska, M. and Kelly, G. "The failure mechanisms of micro-scale cantilevers under shock and vibration stimuli”, Strain, 45(3), pp. 283-294 (2009).

13. Kimberley, J., Cooney, R., Lambros, J., Chasiotis, I. and Barker, N. "Failure of Au RF-MEMS switches subjected to dynamic loading", Sensors and Actuators A: Physical, 154(1), pp. 140-148 (2009).

14. Srikar, V. and Senturia, S.D. "The reliability of microelectromechanical systems (MEMS) in shock environments", Journal of Microelectromechanical Systems, 11(3), pp. 206-214 (2002).

15. Li, G. and Shemansky, F. "Drop test and analysis on micro-machined structures", Sensors and Actuators A: Physical, 85(1), pp. 280-286 (2000).

16. Krylov, S. and Maimon, R. "Pull-in dynamics of an elastic beam actuated by continuously distributed electrostatic force", Journal of Vibration and Acoustics, 126(3), pp. 332-342 (2004).

17. Krylov, S. "Lyapunov exponents as a criterion for the dynamic pull-in instability of electrostatically actuated microstructures", International Journal of Non-Linear Mechanics, 42(4), pp. 626-642 (2007).

18. McCarthy, B., Adams, G.G., McGruer, N.E. and Potter, D. "A dynamic model, including contact bounce, of an electrostatically actuated microswitch", Journal of Microelectromechanical Systems, 11(3), pp. 276-283 (2002).

19. Younis, M.I., Modeling and Simulation of Microelectromechanical Systems in Multi-Physics Fields, Citeseer (2004).

20. Moghimi Zand, M. and Ahmadian, M. "Vibrational analysis of electrostatically actuated microstructures considering nonlinear effects", Communications in Nonlinear Science and Numerical Simulation, 14(4), pp. 1664-1678 (2009).

21. Nayfeh, A.H. and Younis, M.I. "A new approach to the modeling and simulation of flexible microstructures under the effect of squeeze-film damping", Journal of Micromechanics and Microengineering, 14(2), p. 170 (2003).

22. Younis, M.I. and Nayfeh, A.H. "Simulation of squeezefilm damping of microplates actuated by large electrostatic load", Journal of Computational and Nonlinear Dynamics, 2(3), pp. 232-241 (2007).
23. Tajalli, S., Moghimi Zand, M. and Ahmadian, M. T., "Effect of geometric nonlinearity on dynamic pullin behavior of coupled-domain microstructures based on classical and shear deformation plate theories", European Journal of Mechanics-A/Solids, 28(5), pp. 916-925 (2009).

24. Lotfi, M., Moghimi Zand, M., Isaac Hosseini, I., Baghani, M. and Dargazany, R. "Transient behavior and dynamic pull-in instability of electrostaticallyactuated fluid-conveying microbeams", Microsystem Technologies (In press).

25. Moghimi Zand, M. and Ahmadian, M.T. "Dynamic pull-in instability of electrostatically actuated beams incorporating Casimir and van der Waals forces", Proceedings of the Institution of Mechanical Engineers, Part C: Journal of Mechanical Engineering Science, 224(9), pp. 2037-2047 (2010).

26. Moghimi Zand, M., Ahmadian, M.T. and Rashidian, B. "Semi-analytic solutions to nonlinear vibrations of microbeams under suddenly applied voltages", Journal of Sound and Vibration, 325(1), pp. 382-396 (2009).

27. Isaac Hosseini, I., Moghimi Zand, M. and Lotfi, M. "Dynamic pull-in and snap-through behavior in micro/nano mechanical memories considering squeeze film damping", Microsystem Technologies (In Press).

28. Mojahedi, M., Moghimi Zand, M. and Ahmadian, M.T. "Investigation of the Oscillatory Behavior of Electrostatically-Actuated Microbeams", in ASME 2010 International Mechanical Engineering Congress and Exposition, pp. 619-626 (2010).

29. Daneshpajooh, H. and Moghimi Zand, M. "Semianalytic solutions to oscillatory behavior of initially curved micro/nano systems", Journal of Mechanical Science and Technology, 29(9), pp. 3855-3863 (2015).

30. Moghimi Zand, M. and Ostadi Moghaddam, A. "Pull-in instability and vibrations of a beam microgyroscope", Journal of Applied Mechanics, 45(1), pp. 29-34 (2014).

31. Mojahedi, M., Moghimi Zand, M. Ahmadian, M. and Babaei, M. "Analytic solutions to the oscillatory behavior and primary resonance of electrostatically actuated microbridges", International Journal of Structural Stability and Dynamics, 11(06), pp. 1119-1137 (2011).

32. Moghimi Zand. M. and Ahmadian, M.T. "Application of homotopy analysis method in studying dynamic pull-in instability of microsystems", Mechanics Research Communications, 36(7), pp. 851-858 (2009).

33. Alipour, A., Moghimi Zand, M. and Daneshpajooh, H. "Analytical solution to nonlinear behavior of electrostatically actuated nanobeams incorporating van der Waals and Casimir forces", Scientia Iranica, 22(3), pp. 1322-1329 (2015). 
34. Suhir, E. "Could shock tests adequately mimic drop test conditions?", Journal of Electronic Packaging, 124(3), pp. 170-177 (2002).

35. Moghimi Zand, M., Rashidian, B. and Ahmadian, M. "Contact time study of electrostatically actuated microsystems", Scientia Iranica. Transactions B, Mechanical Engineering, 17(5), p. 348 (2010).

36. Bao, M., Yang, H. Sun, Y. and French, P.J. "Modified Reynolds' equation and analytical analysis of squeezefilm air damping of perforated structures", Journal of Micromechanics and Microengineering, 13(6), p. 795 (2003).

37. Huang, J.-M., Liew, K. Wong, C. Rajendran, S., Tan, M. and Liu, A. "Mechanical design and optimization of capacitive micromachined switch", Sensors and Actuators A: Physical, 93(3), pp. 273-285 (2001).

38. Reddy, J.N., Mechanics of Laminated Composite Plates and Shells: Theory and Analysis: CRC press (2004).

39. Younis, M.I., Alsaleem, F. and Jordy, D. "The response of clamped-clamped microbeams under mechanical shock", International Journal of Non-Linear Mechanics, 42(4), pp. 643-657 (2007).
40. Yagubizade, H. and Younis, M.I. "The effect of squeeze-film damping on the shock response of clamped-clamped microbeams", Journal of Dynamic Systems, Measurement, and Control, 134(1), p. 011017 (2012).

\section{Biographies}

Javid Abderezaei received his BS degree in Mechanical Engineering from Khajeh Nasir Toosi University of Technology, Tehran, Iran, in 2014, and is currently an MS degree student in the Department of Mechanical Engineering at University of Tehran, Iran. His research interests include MEMS, BioMEMS, and cell and tissue engineering.

Mahdi Moghimi Zand is a Faculty Member in the Department of Mechanical Engineering at University of Tehran, Iran. He has received numerous academic awards, including the Young Professor Grant and PhD Fellowship of the National Elite Foundation. Dr. Moghimi Zand's research is multi-disciplinary and includes vibrations, MEMS, bio-medical engineering, and cancer engineering. 